\title{
PNEUMONIA IN THE NEWBORN RESULTING FROM THE INHALATION OF GASTRIC CONTENTS
}

\author{
BY
}

\author{
KENNETH RHANEY, M.B., CH.B., and AGNES R. MACGREGOR, M.D., F.R.C.P.Ed. \\ (From the Department of Child Life and Health and the Department of Pathology, University of \\ Edinburgh)
}

In the course of performing post-mortem examinations in cases of neonatal death, we have noted the occurrence of pneumonia with certain special features, which we believe to be caused by the aspiration of stomach contents.

An extensive survey of the available literature published since 1920 revealed that little attention has been devoted to this condition. It was briefly described by one of us (Macgregor, 1939), and various other authors have recognized its occurrence, but an adequate description of the pathological features of the disease in infants or children was not discovered. The condition in adults has been described fully by Irons and Apfelbach (1940), and by Apfelbach and Christianson (1937).

A review of autopsy material at the Simpson Memorial Maternity Pavilion was undertaken, and it was discovered that this type of pneumonia is by no means infrequent.

The following is a review of the pathological features of forty-one cases that occurred during the period 1939-46 inclusive, with an analysis of the clinical and autopsy data.

\section{Morbid Anatomy}

Macroscopical features. The trachea and main bronchi showed intense congestion of the mucous membrane and mucopurulent exudate in the lumen. Foreign material was recognized only in early cases. In those of longer standing the material was obscured by inflammatory exudate or had been aspirated into the intrapulmonary bronchi.

The hungs showed widespread consolidation, which was most extensive and advanced in the bases and posterior borders. Areas of haemorrhage, sometimes very extensive, were invariably present. The cut surface was dark red, moist and friable, and oozed blood. Beads of pus, and sometimes recognizable vomitus, exuded from the cut ends of the intrapulmonary bronchi. Small areas of suppuration were occasionally visible, but these were more frequently found on microscopical examination. Post-mortem digestion of tissue was sometimes evident. Vesicular and interstitial emphysema were rarely observed. Pleurisy was unusual, except in the presence of underlying suppuration. When present, the pleural exudate was scanty and localized to small areas, frequently at the base. It could be wiped off without difficulty and the underlying surface looked normal. Gross empyema was found only when a lung abscess had ruptured through the pleura.

In many cases, although the macroscopical characters suggested the presence of this type of pneumonia, they were not sufficiently distinctive to permit of positive diagnosis without microscopical examination.

Microscopical features. The foreign material in the bronchi was heavily infected with bacteria, and the bronchial walls showed a severe inflammatory reaction (Plate IVa and b). Their surface was usually denuded of epithelium. The wall was severely congested and densely infiltrated with polymorph leucocytes. Purulent exudate was present in the lumen. Aspirated material in the alveoli excited a similar intense inflammatory reaction. This was invariably accompanied by massive haemorrhage, which was responsible for a large part of the consolidation and caused it to be confluent over wide areas.

Under low magnification, the architecture of the lung tissue in the vicinity of deposits of aspirated material was difficult to define because of digestion of the bronchial and alveolar walls and of their content of polymorph leucocytes and red blood corpuscles. The collagen and muscle fibres of the bronchial walls stained feebly with eosin, and the walls of the affected alveoli were also feebly stained. The nuclei of cells in the bronchial and alveolar walls, and in the inflammatory exudate, were pyknotic or had disappeared. A most striking feature was lysis of the red blood corpuscles in the exudate (Plate IVc). These had lost their haemoglobin, and the envelope of the corpuscles stained feebly with eosin, presenting a striking contrast with the unaffected cells in surrounding parts (Plate IVd). If sufficient time had elapsed between death and autopsy, the blood in the alveolar and bronchial capillaries also showed lysis. The changes resulting from digestion and lysis were related to the masses of aspirated material, and were less widespread than the inflammatory exudate or haemorrhage. 
In the most advanced cases, foci of suppuration, which were sometimes confluent, developed in and around bronchi, causing complete disorganization of the bronchial wall and related alveoli. In such cases the development of a septic lymphangitis caused the spread of suppuration along the interlobular septa and by the peribronchial and perivascular lymphatic paths.

In certain cases, routine sections of pneumonic lungs showed the features of an established neonatal bronchopneumonia, with, in addition, small areas of recent aspiration pneumonia. It was usually easy to determine the secondary or terminal nature of these foci resulting from aspiration.

\section{Bacteriology}

Unfortunately, owing to technical difficulties caused by the 1939-45 war, bacteriological investigations could be undertaken in only a few cases. In nearly all the cases that were examined, cultures of Bacillus coli were obtained, frequently in association with indifferent streptococci, and occasionally with Monilia albicans. In a number the results were rejected because control smears of the lung juice failed to indicate satisfactorily that the organisms obtained in cultures were present during life (Macgregor, 1939).

\section{Types of Pneumomia Excluded from this Study}

We have not included in this study cases in which material from the stomach had been aspirated into the respiratory passages shortly before death and had produced no gross or microscopical evidence of any effect on the lungs.

We have also excluded cases of staphylococcal pneumonia, in which suppuration frequently occurs and is usually more extensive and of more rapid development than in the cases under consideration. Although haemorrhage and necrosis are typical features, digestion of tissue does not occur, and lysis of blood is only occasionally seen. Staphylococcus aureus is usually the only organism present, and it is therefore unlikely that this type of pneumonia is the result of aspiration of stomach contents, which contain a variety of bacteria. It is more likely to be caused by aspiration from the mouth or pharynx of food or secretion heavily infected with staphylococci.

Cases of aspiration pneumonia caused by congenital atresia of the oesophagus with tracheooesophageal fistula have also been excluded.

\section{Incidence}

During the years 1939-46 inclusive, 962 autopsies were performed on infants born alive who died in the Simpson Memorial Maternity Pavilion, Royal Infirmary, Edinburgh. Of these, 939 died within one month of birth. Among these there were forty-one cases of pneumonia resulting from the aspiration of gastric contents. Thirty-nine of the infants were less than one month old. In thirty-six cases the pneumonia was believed to be caused entirely by aspiration. In the remaining five it was thought that pneumonia had existed before aspiration occurred, because the lungs showed established bronchopneumonia, complicated by small areas of recent aspiration pneumonia.

A remarkable increase in the incidence of aspiration pneumonia was observed during the last two years of the period. During the six years 1939-44, when 731 autopsies on infants born alive were performed, fourteen cases occurred (1.9 per cent.). During the two years 1945-46, when 252 autopsies were performed, twenty-seven cases occurred (10.7 per cent.).

\section{Clinical Fentures}

We have found no evidence that this type of pneumonia differs in its clinical characters from other types which occur during the neonatal period, and diagnosis can only be established with certainty by pathological examination. In only one of the forty-one cases was a definite diagnosis made because the occurrence of aspiration was observed, and was followed by the development of severe and rapidly fatal bronchopneumonia.

\section{Incidence in Rehation to Birth Weight}

In fig. 1, incidence is plotted in relation to birth weight, the ordinate indicating the number of cases, and the abscissa the birth weight. The standard of prematurity applied was the accepted one: infants of $5 \frac{1}{2} \mathrm{lb}$. and under at birth were regarded as premature. A vertical line drawn at $5 \frac{1}{2} \mathrm{lb}$. separates the mature on the right from the premature on the left. Thirty-three of the infants were premature, and of these twenty-four were under $4 \mathrm{lb}$. in weight.

\section{Incidence in Relation to Age at Denth}

In fig. 2 the ordinate represents the number of cases and the abscissa the age at death in days. Twenty-five of the infants died between the fourth and ninth day.

\section{Matermal Health during Pregnancy}

In twenty-six of the cases pregnancy had been normal; in seven the mother suffered from preeclamptic toxaemia, in one from hypertension, in another from chronic cardiac disease, and in one the mother's blood Wassermann reaction had been positive. Five infants were admitted to hospital after birth, and no details of the maternal history were available.

\section{Type of Delivery}

Delivery was spontaneous, either vertex or breech, in twenty-five cases. Four breech extractions required assistance. Ten infants required forceps delivery, and two deliveries were by Caesarian section. 


\section{Condition of the Infont at Birth}

An attempt to correlate the condition of the infants at birth with the occurrence of aspiration of stomach contents proved difficult. Because of

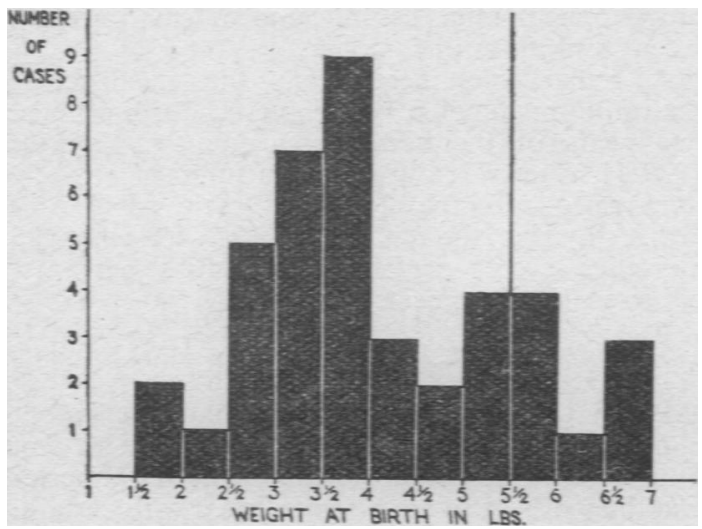

Fig. 1. Incidence in relation to birth weight, 41 cases.

shortage of staff caused by the war, the recorded information was inadequate in most instances, and the cases in which information was available were too few to allow any conclusions to be drawn from them. It was noteworthy that most of the infants who died during the first four days were in an unsatisfactory condition at birth.
Five infants suffered from thrush infection: in two it was confined to the oral cavity; in three the oesophagus was also involved. In one case there was peptic ulceration of the lower end of the oesophagus.

Ten infants were known to have vomited. In one case the vomiting occurred more than two weeks before death and was probably not responsible for the pulmonary condition, but in the other nine the vomiting may have been of importance in the etiology of the pneumonia.

\section{Type of Feeding}

Only six infants were entirely breast-fed; fourteen received expressed breast milk only; eight received breast milk and complementary feeds, and eleven infants received artificial feeds. Three of the infants were fed by oesophageal tube. In two cases no details of the method of feeding were available.

\section{Discussion}

The description of ' aspiration pneumonia' by Irons and Apfelbach (1940) corresponds closely to the condition we have described in the newborn. These authors call attention to the characteristic features of the microscopical picture, noting that - the tissues removed from the thorax have lost their capacity for distinct staining in contrast to tissues removed from other portions of the body, indicating a disproportionate degree of post-mortem change

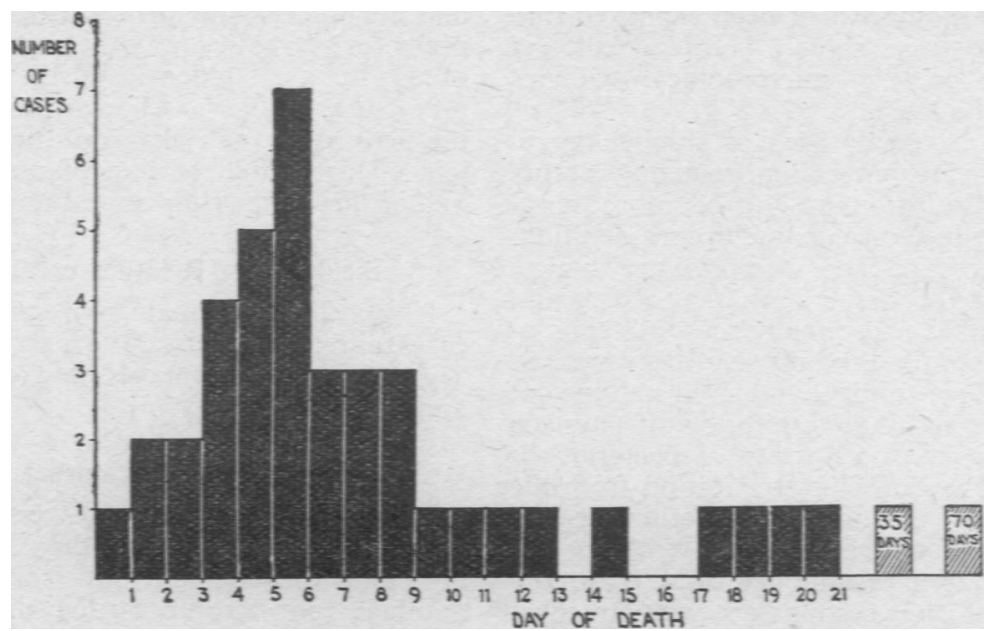

Fig. 2-Incidence in relation to age at death, 41 cases.

\section{Associated Conditions in the Irfant}

Three infants had developmental abnormalities: one was a mongol, another suffered from hydrocephalus with meningocele, and the third infant had atresia of the duodenum.

Eighteen infants suffered from intracranial haemorrhage, and in nine of these the condition was severe. in the lungs. The erythrocytes in the alveolar spaces, and often those in the capillaries, have lost a large amount of their hemoglobin so that they stain only as shadowy outlines.' They emphasize the importance of this, "because in instances of pneumonia caused by the usual respiratory organisms hemolysis and post-mortem change are not characteristic of the alterations in lung tissue. 


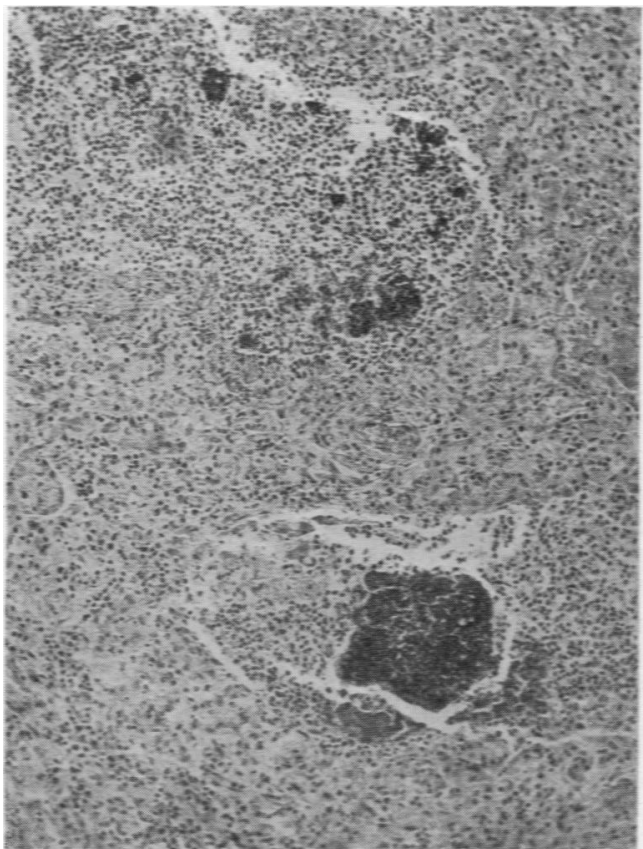

(a).-Bronchi containing masses of aspirated material and showing acute inflammation which obscures their walls. Haematoxylin and eosin. $\times 100$.

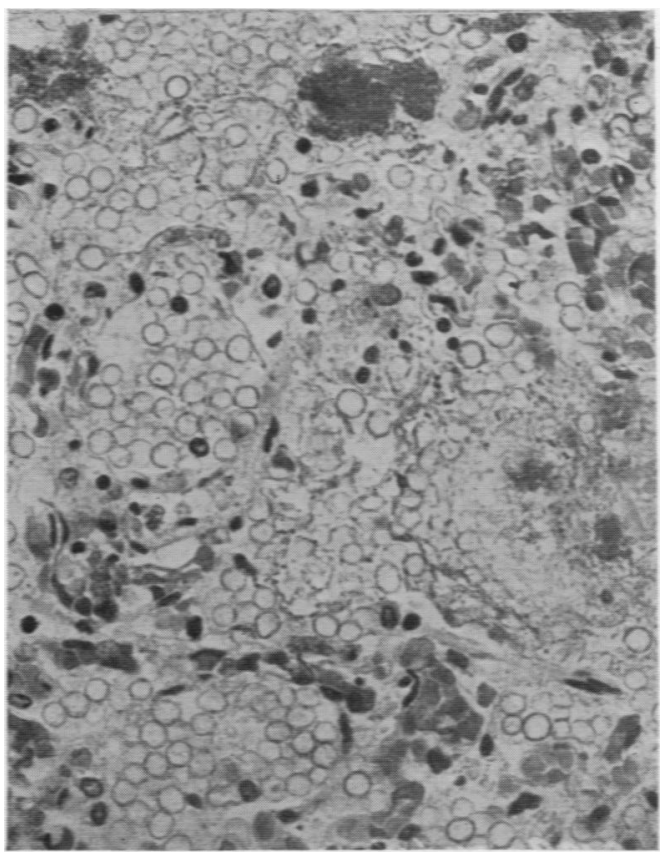

(c).-Lysed red blood corpuscles in alveoli; the lysis is in relation to deposits of aspirated material. Haematoxylin and eosin. $\times \mathbf{4 0 0}$.

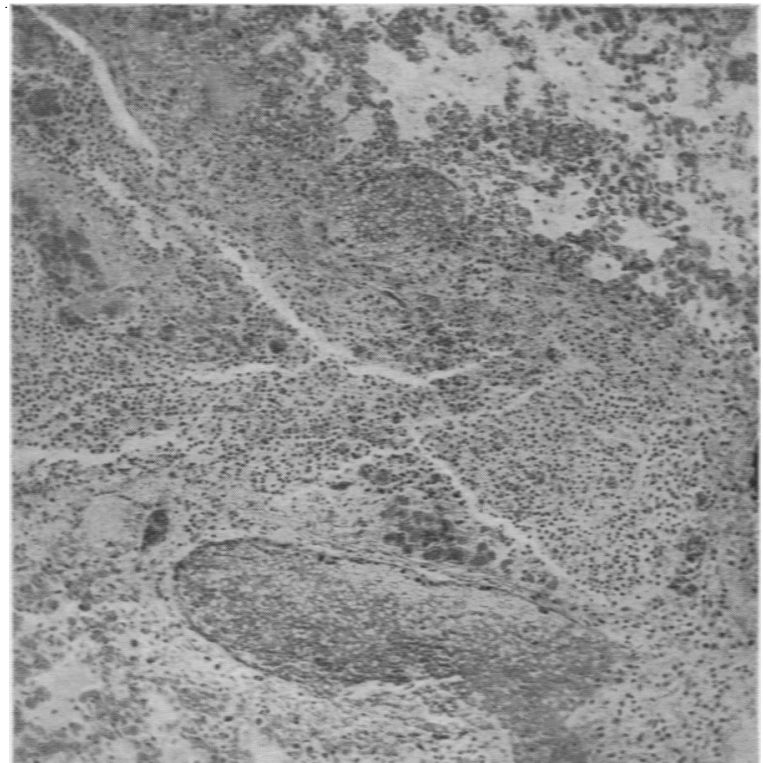

(b).-A bronchus which contains aspiraled material and shows incipient suppuration. An adjacent vein shows post-mortem lysis of erythrocyles on the side nearer the bronchus. Haematoxylin and eosin. $\times 100$.

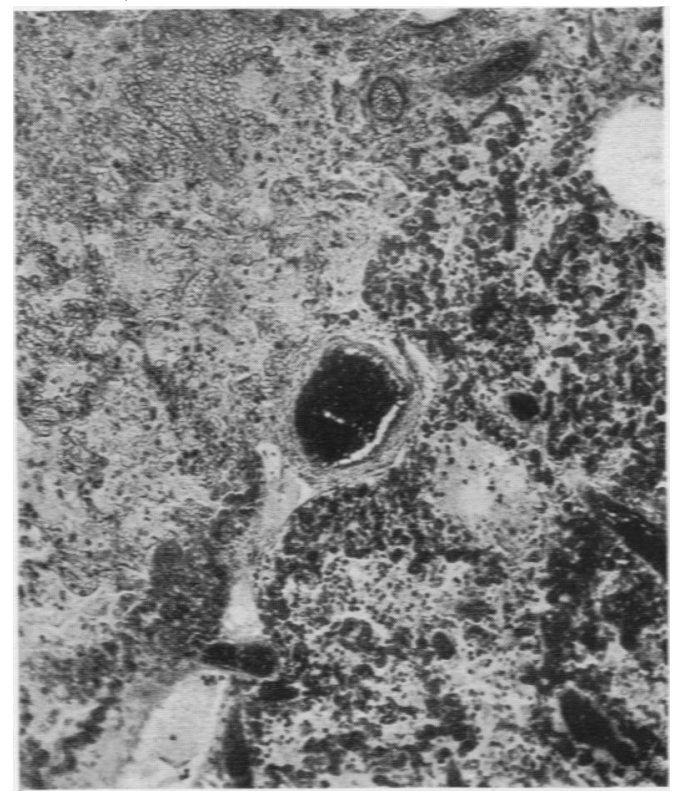

(d).- Illustrates an area of haemorrtage and pneumonia in part of which lysis and digestion have occurred. Haematoxylin and eosin. $\times 100$. 
... Thus, advanced changes in the lungs indicate that material has been introduced into them that can accelerate post-mortem alterations.' In the opinion of Irons and Apfelbach digestion of tissues and lysis of blood occur only after death. They stressed the importance of performing autopsies within an hour or two of death, because the postmortem changes produced by the aspirated material are then more readily recognized than when generalized post-mortem changes have occurred. In most of our cases it was not possible to perform the autopsy so promptly, and on histological examination the blood in the alveolar capillaries had usually undergone lysis. This had obviously occurred after death, but as an aid to diagnosis it was nevertheless significant, as it occurred only in areas related to aspirated gastric contents.

Irons and Apfelbach introduced stomach contents, in which there was no free hydrochloric acid, into the bronchial tree of a dog and produced 'the same types of haemorrhagic, hyperaemic and oedematous alterations in the lung . . . as are observed in persons in whom the pathologic changes interpreted as aspiration bronchopneumonia are found at necropsy.'

Apfelbach and Christianson (1937) found that the introduction into the lungs of animals of 'gastric fluid containing normal amounts of hydrochloric acid usually did not produce death, but a temporary hyperemia of the lungs occurred. Also stomach content free of hydrochloric acid and containing bacteria, when passed through a Berkefeld filter, also produced only a transitory hyperemia of the lungs. Death occurred in animals only if stomach content containing bacteria was used.' (Irons and Apfelbach, 1940).

Miller (1941) showed that the gastric acidity of normal full-time infants is usually high at birth but falls during the first ten days of life and thereafter gradually rises. In premature infants the gastric acidity is much lower during this period. In view of these observations it seems possible that hyperaemia induced by the inhalation of gastric contents containing free hydrochloric acid might be a factor in the production of the massive pulmonary haemorrhage that was observed in many of these infants. This haemorrhage sometimes caused death before the development of much inflammatory reaction. In the absence of free hydrochloric acid, effects would depend to a greater extent on bacterial growth and would therefore be slower to develop (Simonds, 1940).

Frank vomiting to which aspiration could be attributed was recorded in a minority of cases. We believe that regurgitation, to which less attention may be paid, is also of great importance. It may occur without being noticed by the attendants, and, unless regurgitated material escapes from the mouth and stains the infants' clothes, there may be nothing to indicate that it has occurred. There is a great risk that regurgitation may occur during feeding unless adequate care is being taken.
Aspiration of material from the mouth will be favoured by insensitivity of the protective reflexes of the pharynx and respiratory tract. Prematurity, feebleness, deep sleep and narcosis, intracranial haemorrhage, and chilling are some of the factors that are known to depress the activity of these reflexes (Amberson, 1937; Nungester and Klepser, 1938). Prematurity is often associated with several other of these factors, and this probably explains the high incidence of aspiration pneumonia in premature infants. In our series several infants died of aspiration pneumonia within four days of birth. We believe that there is a grave risk of aspiration of gastric contents when infants are fed while still suffering from the effects of birth stress or maternal narcosis.

We have called attention to the striking increase in the incidence of aspiration pneumonia during the last two years of the period under survey. It would be unwise to draw dogmatic conclusions from this observation, but during these two years the work of the hospital greatly increased without a corresponding increase in the nursing staff. Under such circumstances the amount of time and care devoted to each infant would of necessity be reduced. Further observation at a later time will be required to show that improved conditions of staffing bring about a reduction in the incidence of aspiration pneumonia.

\section{Summary}

A review of forty-one cases of pneumonia resulting from the inhalation of gastric contents is presented.

The main pathological features are extensive consolidation with haemorrhage and a tendency to suppuration. Microscopically, digestion of tissues and lysis of blood in relation to aspirated material are characteristic.

An analysis of the clinical data is given.

Attention is drawn to predisposing factors with particular reference to prematurity.

An increase in the incidence during the last two years of the period under review was observed. It is suggested that this may be related to a shortage of nursing staff.

We wish to express our thanks to Professor Charles McNeil, Professor R. W. B. Ellis, and Dr. J. L. Henderson for helpful criticism and access to clinical records.

\section{REFERENCES}

Amberson, J. B. (1937). Inter. Clin., 3, 126.

Apfelbach, C. W., and Christianson, O. O. (1937). J. Amer. med. Ass., 108, 503.

Irons, E. E., and Apfelbach, C. W. (1940). J. Amer. med. Ass., 115, 584.

Macgregor, A. R. (1939). Arch. Dis. Childh., 14, 323. Miller, R. A. (1941). Arch. Dis. Childh., 16, 22.

Nungester, W. J., and Klepser, R. G. (1938). J. infect. Dis., 63, 94.

Simonds, J. P. (1940). J. Amer. med. Ass., 115, 587. 